\title{
Simulation Study of Topological Structures and Node Coordinations for Deterministic WSN with TSCH
}

\author{
Vijaya Perumalla\#, B. Seetha Ramanjaneyulu\#, Ashok Kolli" \\ \# Department of ECE, VFSTR University, Vadlamudi, Guntur, 522213, India \\ E-mail:vijayaperumalla1@gmail.com,ramanbs@gmail.com,ashok.kolli13@gmail.com
}

\begin{abstract}
Time-Slotted Channel hopping (TSCH) that was introduced in IEEE802.15.4e is a promising technique to offer deterministic data deliveries in Wireless Sensor Networks (WSN). Its main strength lies in using multiple channels for the transmissions, using frequency hopping method. However, it takes more time for initializing the network, as beacons have to be released on multiple frequencies and scanning needs to be carried out by the devices in all those frequencies for joining the network. In this work, a simulation study is carried out to investigate the effect of these delays for different types of multihop networks. The benefit of introducing multiple coordinators in reducing these time delays is also studied. It is found that the delays are increasing linearly with the number of hops in the network and adding an additional coordinator can bring down the initialization time by half.
\end{abstract}

Keywords - TSCH, WSN, Enhanced Beacon, Network Coordinator, Channel Hopping Sequence Length.

\section{INTRODUCTION}

Collecting the field data from the senors is an important and crucial task in industrial automation. In the early days of automation, every sensor was connected to the controller with its unshared cable. Communication bus technologies were introduced in 1990s which made it possible to remove the bundles of cables with few shared bus cables. Then came the introduction of wireless communication systems, with which cables could be removed completely [6]. These implementations gained popularity with the Industrial environment being integrated with the Internet world these days, to meet the growing demands of process efficiencies and the financial objectives [4].

The industrial applications often require reliability and security because the quality and efficiency of goods and worker safety are based on these networks. In this aspect, it is found that wireless transmissions are not as reliable and deterministic as the wired communications. Interference is the main problem to wireless transmissions. This problem is more acute in unlicensed bands. In general, unlicensed ISM bands are used for the transmissions of wireless sensors. In such cases, it may happen that the sensor that is trying to send a crucial data also may not get the channel at the needed instant of time. Hence wire-like reliability is to be incorporated into these wireless data acquisition systems.
In order to obtain the deterministic services for industrial automations [12], TSCH protocol is introduced as part of IEEE802.15.4e standard [10]. It uses frequency hopping method, in which different transmission frequencies are chosen at different times by the nodes of WSN. However, there occurs some delay in initializing the network in this method. It is analyzed in this work the impact of the delays in forming the network, by finding the time delays of 1-hop 2hop and 3-hop architectures of the network. The effect of introducing additional coordinators which can initiate the network formation is also found.

In section-2 of the paper, the deterministic need of sensor transmissions are discussed. Section-3 is about TSCH mechanism, and section-4 deals with the design and implementation of WSN with TSCH mechanism along with the simulation results of the time dealys of the multihop network formations. Adding additional coordinators is also included here. Section-5, concludes the paper.

\section{DETERMINISM}

The reduced installation and maintenenace costs, and the possibility of placing the sensor at inaccesssible points are the attractive features of adopting wireless communications in automation, in the place of wired buses [11], [13]. Hence the deployment of wireless sensor networks is increasing in industries because of its suitability in places inaccessible by 
wired technology [6] and the capability of creating spontaneous, pervasive, flexible and energy efficient networks and the detection of possible system failure [1].

Network formation is an important aspect of TSCH which is an indication of determinism i.e.;lower the joining time higher is the determinism. To provide deterministic services, TSCH Protocol is adopted in industrial low-power wireless standards such as Wireless HART which is an International standard in which there is no guarantee for the end-end deadline of its critical traffic[8] and ISA100.11a (International Society of Automation) which is useful for the noncritical monitoring[9] and as a part of IEEE 802.15.4e

In $\mathrm{TSCH}$, the network formation starts by electing one mote as a coordinator and the coordinator broadcasts Enhanced Beacon (EB) frames. If a not yet synchronized node receives an $\mathrm{EB}$, it records the $\mathrm{ASN}$ and synchronize itself to the network. The synchronized nodes start sending EBs for extending network coverage. The channel hopping nature takes a longer time for the synchronization of all the nodes to the network i.e.; the coordinator and the new joining motes need to be aligned on the same frequency. When an EB is sent by the coordinator on a given channel the motes not yet synchronized could be listening another channel. Thus the time taken for the motes to start transmissions gets slower [1].

\section{TIME SLOTTED CHANNEL HOPPING (TSCH)}

IEEE802.15.4-2006 is the standard for the physical and MAC layer functioning of personal area network, like Zigbee. It face some limitions like Unbounded Delay, Less communication reliability and less protection for interferences/fading, Powered relay nodes.

IEEE802.15.4e was developed improving MAC layer to improve the deterministic needs of industrial applications and to define multi-ring networks. TSCH is one of the MAC behavior modes of IEEE 802.15.4e[2].

\section{A. Time-Slotted Channel Hopping (TSCH)}

TSCH Protocol originated from TSMP (Time Synchronized Mesh Protocol) offers $99.999 \%$ reliability in MAC layer, improves the automation process, efficiency and productivity in industries by offering predictable latency, improved network capacity, maximum reliability and maintains very low duty cycles[5].

TSCH is a combination of Time Slotted Access with MultiChannel and Channel hopping Capacities. Time slotted nature provides deterministic latency by eliminating the collision among competing motes. Multi-channel nature improves the network capacity by allowing more nodes to exchange the data frames in the same timeslot using different channel offsets. Channel hopping nature helps in improving the reliability by lowering the effects of multipath fading and interference [2].

$\mathrm{TSCH}$ is topology independent and suitable for multihop networks[7] because the frequency hopping nature makes efficient use of the available resources.

Fig.1 shows the network with different transmissions and receptions between the motes and the transmissions are scheduled using two cycles each containing 3 sloframes satisfying the TSCH features such as time slotted access i.e $\mathrm{D} \rightarrow \mathrm{C}$ and $\mathrm{A} \rightarrow \mathrm{C}$ used different time slots and the channel hopping nature i.e $\mathrm{B} \rightarrow \mathrm{A}$ and $\mathrm{G} \rightarrow \mathrm{D}$ transmissions and the multi channel communication mechanism i.e $\mathrm{F} \rightarrow \mathrm{D}$ and $\mathrm{E} \rightarrow \mathrm{C}$ transmitting in the same time slot using different channel offsets. TSCH schedule also uses shared cells apart from dedicated cells i.e the transmissions $\mathrm{H} \rightarrow \mathrm{E}$ and $\mathrm{I} \rightarrow \mathrm{E}$ takes place in the same time slot and in the same frequency and the collisions in shared cells are avoided using the random back off algorithm. EB's are scheduled to advertise in the timeslot 0 and channel offset 0 . The frequencies taken will be repeated with the no. of timeslots considered in a slotframes. The transmissions scheduled are repeated after every cycle and hops the channel in every cycle i.e.; $\mathrm{B} \rightarrow \mathrm{A}$ in first cycle uses $\mathrm{Ch} 20$ and $\mathrm{B} \rightarrow \mathrm{A}$ in second cycle uses $\mathrm{Ch} 15$ based on the links assigned for each scheduled cell.

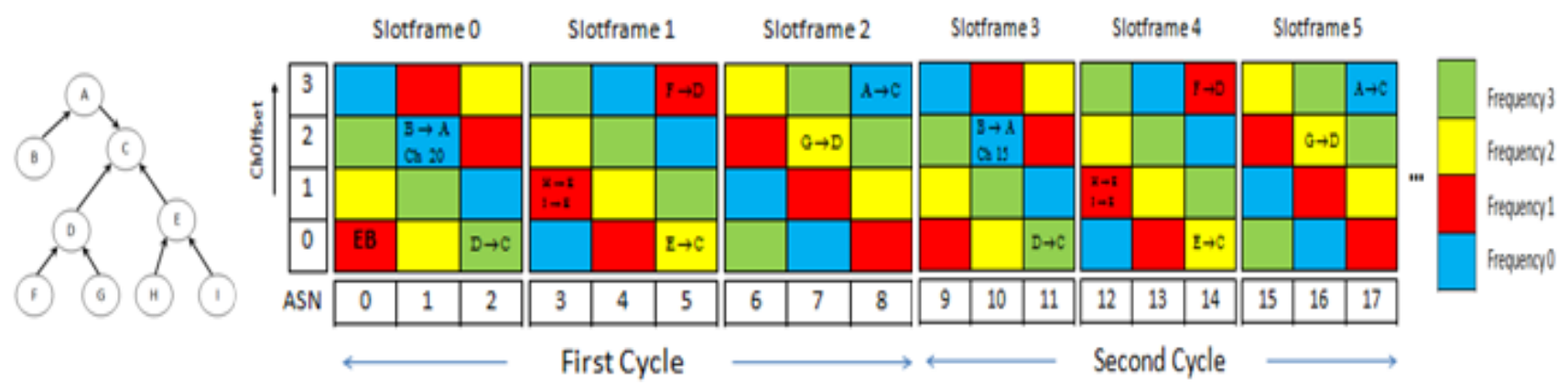

Fig.1 A TSCH Network with a Schedule using different frequencies

The problem with wireless are multi-path fading and external interference. The channel hopping technique smoothens the contribution of each frequency even if some frequencies are busy and causes links and topology to be more stable. The channelOffset is translated into different frequencies at different slotframe cycles. Hence EB frames are sent on all the available frequencies.

$$
\text { frequency }=\mathrm{F}\{(\mathrm{ASN}+\text { channelOffset }) \% \mathrm{n}\}
$$


The join priority field is used to decide on which node to join. The ASN no. increases by 1 for every timeslot and it is encrypted as 5 byte no. for security reasons. the ASN is contained in an IE(Information Element). The no. of slotframes and the no. timeslots in a slotframe are application specific.

If a node is synchronized in a TSCH network, Time is divided into time slots. The duration of timeslot is $10 \mathrm{~ms}$. Single time slot is sufficient for a MAC frame to send from one to another, and to reply with an acknowledgment (ACK) indicating successful reception. Time slots are grouped into slotframes which repeats over a period of time.

In a TSCH network because of its slotted nature, nodes require tight synchronization hence they are equipped with clocks to add the timing information in all packets and helpful for the neighbor nodes in resynchronising to one another whenever they exchange data. For example in Acknowledgment-based synchronization, the sender node is synchronized to the clock of the receiver because it calculates the difference in time between the expected time and the actual time and provides information to the sender node in its acknowledgment.

In the presence of traffic in the network, nodes resynchronize using the data frames they exchange. In the absence of traffic, nodes synchronize to time source of the neighbor periodically but not to drift in time. In the absence of communication for some time, nodes exchange Keep Alive packets to resynchronize.

The TSCH standard defines the following precedence rules in case of multiple slotframes such as transmissions take precedence over receptions and lower slot frame identifiers take precedence over receptions and lower slot frame identifiers take precedence over higher slot frame identifiers [3]
The Design of WSN with TSCH MAC is carried out in Cooja Simulator. The TSCH MAC of $802.15 .4 \mathrm{e}$ is implemented in Contiki OS and its performance evaluations are tested in Cooja.

The WSN network with TSCH MAC is arranged in multiple rings, and it is simulated in Cooja Simulator, to identify the stated problem i.e., more joining time in multiring network

Here the EB packet size considered is 31 bytes with a header length of 12 bytes and for every mote the EBs are advertised in the timeslot id 0, channelOffset 0, Slotframe identifier 0. Since the EBs are advertised the link type is set 1. The coordinator PAN id is abcd and the channels are switched for every one second. The scheduling used is minimal because of the absence of centralized or distributed scheduling algorithms.

Here the long joining time is analyzed by considering two scenarios 1,2

A. Scenario-1: Analysis of joining time in different ring network

The sensor network is created with 31 motes in cooja simulator. The first mote is taken as a coordinator and the remaining 30 motes are arranged in different topologies. The channel hop sequence used is $4 \_4$ i.e $\{15,25,26,20\}$

1) Case 1- One ring topology: In this case, all the 30 motes of Cooja type will be in single ring distance with coordinator centralized as shown in Fig. 2(a). The coordinator starts sending the EBs at a regular interval of time by switching the channels for every one second. Since all the motes are in the transmission range to the coordinator. The motes which are in the same channel at a particular time will get synchronized to the coordinator directly. The motes synchronized (joined) to the network starts sending their own EBs to the motes in its range. In this way all the motes join to the network and the joining time is observed in the mote output of cooja simulator.

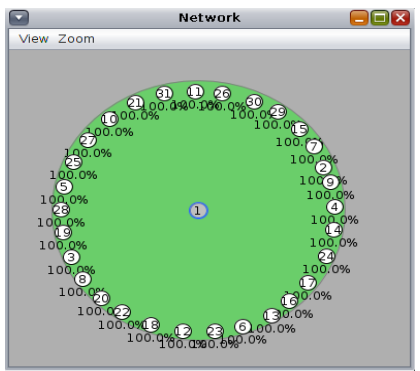

(a)

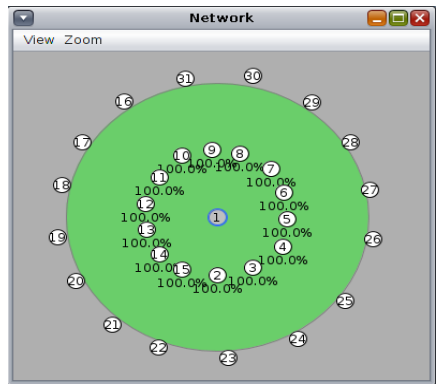

(b)

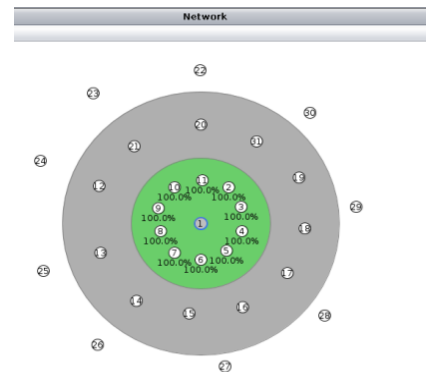

(c) 
2) Case 2- Two Ring Topology: In this case, all the 30 motes of cooja type are arranged in two rings (first 15 motes are in 1-ring and the next 15 motes are in 2-rings distance) with coordinator centralized Fig. 2(b). The motes of the first ring get synchronized to the network first. The mote from first ring which got synchronized start sending its EBs to the motes in its range of the second ring and also to the neigh boring motes of the first ring. In this way, the motes in the second ring starts synchronizing to the network in parallel with the motes of the first ring which are in range with the coordinator. It was observed from the mote output that at $3655 \mathrm{~ms}$ the mote of the first ring ID: 5 and the mote of the second ring ID: 30 got associated to the network. The motes of 2 nd ring have to be communicated through the first ring. Hence the motes of $1^{\text {st }}$ ring get synchronized to the network faster than the motes of the second ring. The joining time of the first ring is less compared to the joining time of the second ring motes from the Fig. 5

3) Case 3-Three Ring Topology: In this case, all the 30 motes of cooja type are arranged in 3- rings (first 10 motes are in 1-ring and the next 10 motes are in 2 ring and the last 10 motes are in the 3-ring distance) with coordinator centralized Fig. 2(c). First one of the 1 st ring motes associate to the network. This mote starts advertising Enhanced beacons to the motes in its range i.e to the neighbour motes in 1ring range and to the motes of 2-ring range. If the channel synchronization happens then the motes of the different rings get associated to the network. On the whole, the joining time of the motes of the first ring will synchronise first to the network compared to the motes of another ring.

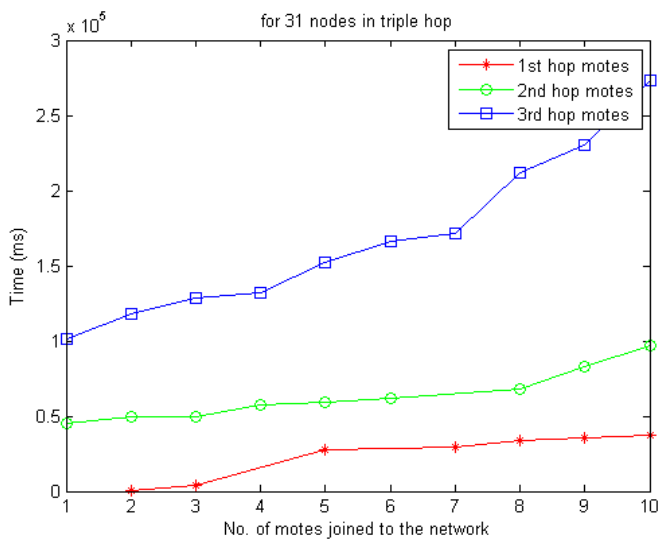

Fig. 3 Analysis of joining time of the motes in different ring topology

In the $1^{\text {st }}$ ring, 2 motes got associated to the network by the time of $505 \mathrm{~ms}$ and the $1 \mathrm{st}$ mote of $2^{\text {nd }}$ ring got synchronized to the network at $45415 \mathrm{~ms}$ and the $1^{\text {st }}$ mote of $3^{\text {rd }}$ ring synchronized to the network at $101515 \mathrm{~ms}$.

In the $1^{\text {st }}$ ring, the time taken for all the 10 motes to get synchronized to the network is $37375 \mathrm{~ms}$. The motes of the second ring get associated to the network by $97,315 \mathrm{~ms}$ and the motes of the third ring have taken a time of $273505 \mathrm{~ms}$ to synchronize to the network. Hence we can say from the Fig. 3 the time taken for the motes of the last ring is more than the motes of $1^{\text {st }}$ ring.

Here the comparative study for all cases of Scenario-1 is made

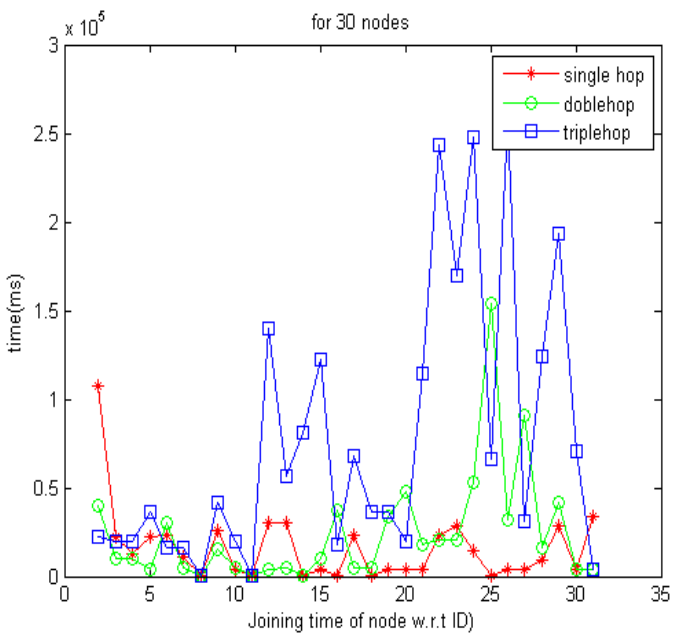

Fig. 4 Analysis of the Joining time of the different ring topologies considered

From the Fig.4 we can observe the joining time of particular mote in each case. $3^{\text {rd }}$-ring cooja motes have taken a longer time compared to $2^{\text {nd }}$-ring WSN and $2^{\text {nd }}$-ring motes have taken much time than $1^{\text {st }}$-ring WSN.

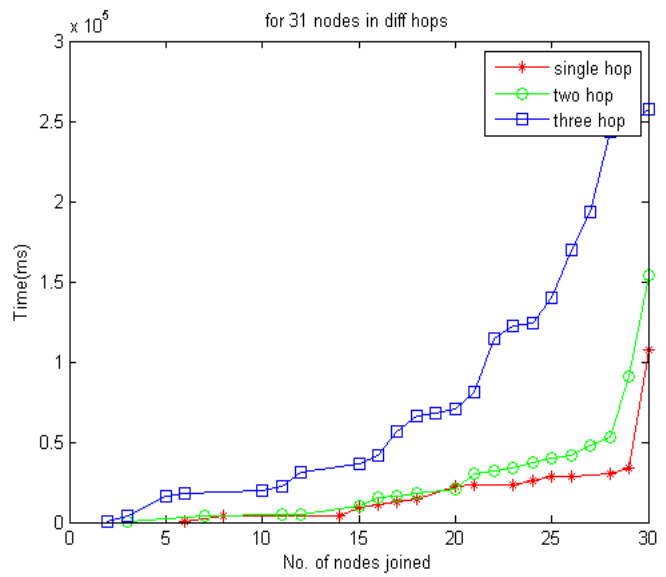

Fig. 5 Analysis of joining times for the total no. of motes joined w.r.t time

Fig.5 shows no. of nodes vs time graph. Here we can clearly identify the different times taken for all 31 motes to associate to network in different ring WSN's. It was found that joining time increases with increasing rings.

The single ring topology motes got synchronized to the network by 1,20,000ms and the double ring topology network got synchronized to the network by at $2,60,000 \mathrm{~ms}$.

Joining time of a mote is based on Mote initialization, Transmission range, Scanning channel, Join priority, Network topology 


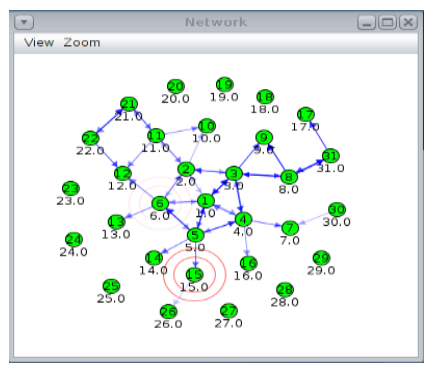

(a)

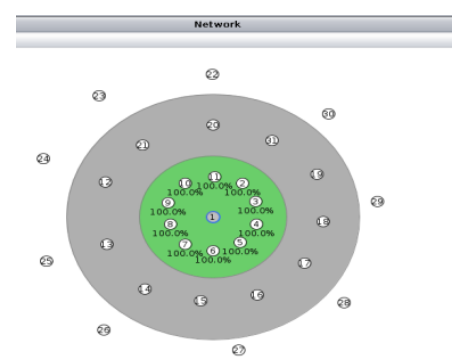

(b)

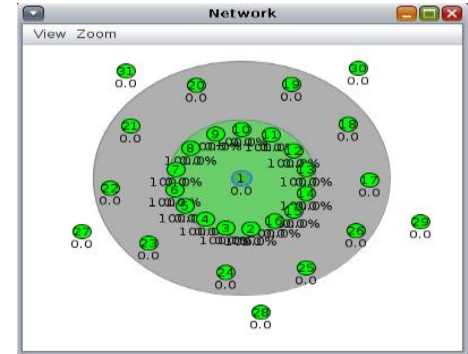

(c)

Fig. 6 A WSN network of (a) 5-10-15 Topology (b) 10-10-10 Topology (c) 15-10-5 Topology

B. Scenario-2: Analysis of different topologies of Multi hop WSN's

This scenario is considered in order to test the reaction of multiring networks to TSCH MAC.

The different ring networks are tested i.e in case-1: The first ring contains less no. and the second ring contains double the no. nodes and triple ring contains triple the no. first ring nodes. In case-2: equal no. nodes are placed in all rings. In case-3: Reverse of case-1 is taken.

1) Case-1:5-10-15: In this arrangement, in the first ring, less no. of motes (5 motes) placed and the second ring 10 motes are used and in the last ring highest no. of 15 motes are taken. The topology considered is shown in Fig. 6(a)

2) Case-2: 10-10-10: Refer to case-3 of scenario-1. Equal no. nodes are placed on the all the rings as shown in Fig. 6(b)

3) Case-3: 15-10-5: This is a reverse case of case-1. First ring contains 15 motes and the second ring contains 10 motes and the third ring contains 5 motes as shown in Fig. 6(c)

All the 3 different scenarios are considered and the grah is plotted as shwn in Fig. 7.

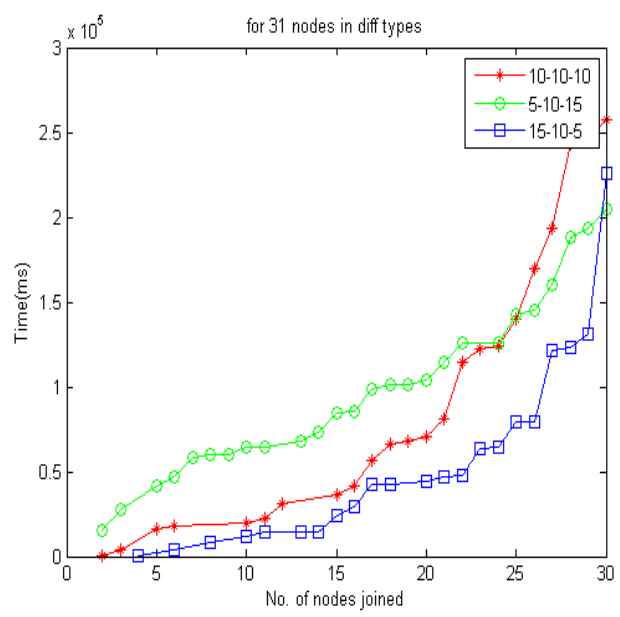

Fig. 7 Analysis of joining time for different WSN topologies considered

Here the joining time of motes in different structured topologies are considered and the difference in time can be observed from the figure and the joining time increases with the no. of rings increased.

From the 2 scenarios, the joining time of the motes in all the topologies is large. Hence a method of increasing the number of coordinators is proposed.

\section{Scenario-3: Increasing the no. of Coordinators}

To reduce the joining time of nodes in the network, it was proposed to increase the no. of nodes involved in sending EB's (coordinator) as shown in Fig. 8.

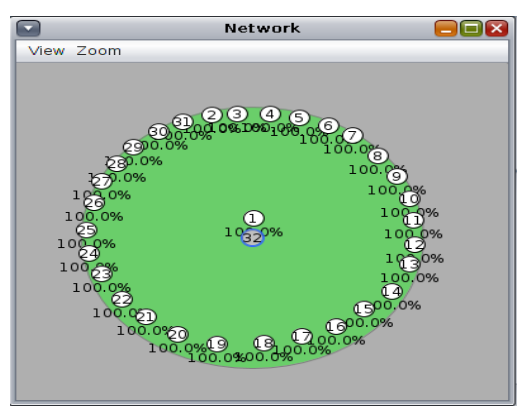

(a)

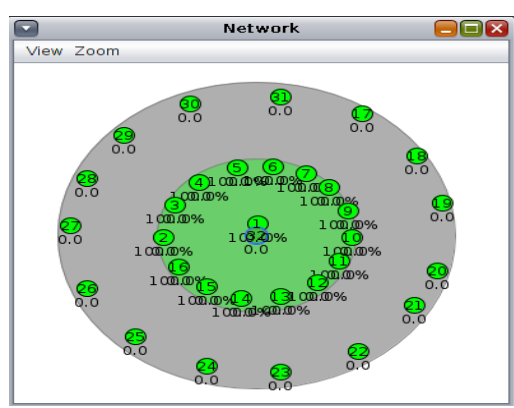

(b)

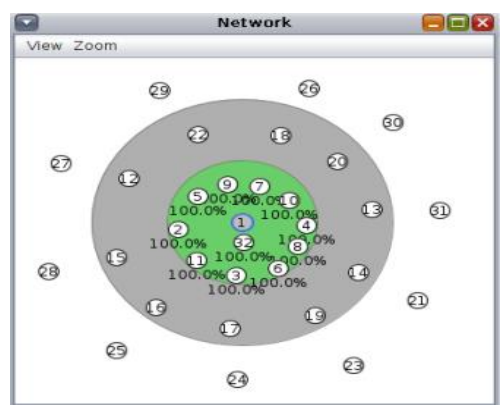

(c)

Fig. 8 (a) One Ring Topology (b) Two Ring Topology (c) Three Ring Topology with two coordinator 


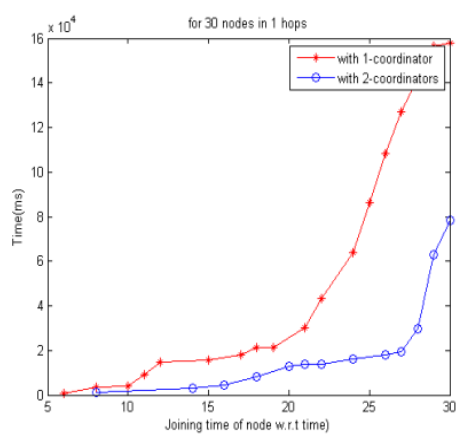

(a)

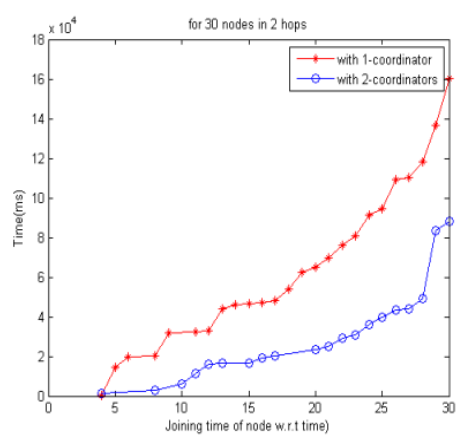

(b)

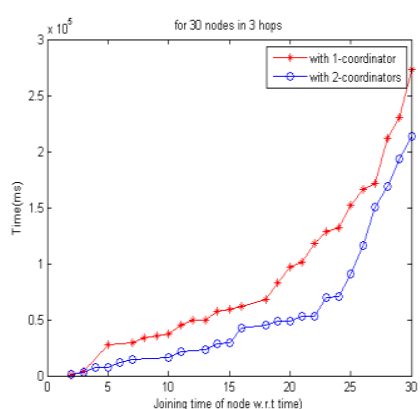

(c)

Fig.9 Analysis with 2 coordinators In (a) single ring topology(b) double ring topology (c) triple ring topology

Since there are 2 coordinators, both will be in different channels sending EBs. Hence the motes listening on the channels to join to the network can be able to listen to the EBs from both the coordinators. The motes which are on the same channel with any of the coordinators join to network. Hence the joining time is reduced because of increased channels.

Fig. 9 shows the joining time of networks with single and double coordinators in single ring topology, double ring topology and triple ring topology. We can observe by the simulation analysis in all different topologies, joining time got reduced. Hence the proposed method of increasing the no. of coordinators is true and this is also helpful in reducing the power as the time to keep its radio channel $\mathrm{ON}$ to receive a valid EB gets reduced because of double coordinators.

\section{Scenario-4: Comparisons on channel hopping sequences}

The default channel hopping sequences of IEEE 802.15.4e are 4_4 and 4_16 and 16_16 such as $\{15,25,26,20\},\{20,26$, $25,26,15,15,25,20,26,15,26,25,20,15,20,25\},\{16,17$, $23,18,26,15,25,22,19,11,12,13,24,14,20,21\}$ respectively.
From the simulation results of Fg. 10, it is advisory to take less no. channels with max. sequence length i.e., the channel hopping sequence 4_16 in order to achieve the less joining time for the synchronization.

\section{CONCLUSION}

A TSCH MAC network with multiple rings are created and the performance analysis for the joining time of the nodes says that the multiple rings network take more time to join the network than the single ring networks.

To reduce the joining time of the WSN network implemented with TSCH, the no. of coordinators are increased and simulation results proved it by taking the less joining time for the synchronization to the network.

Simulation analysis conducted on all different topologies and with the different channel hopping sequences and with different coordinators and observed that the usage of more coordinators gives best results and better use of less no. of channels with more sequence length i.e using 2 cordinators with channel hopping sequence 4_16 gives the best results to TSCH suitable for industrial applications.

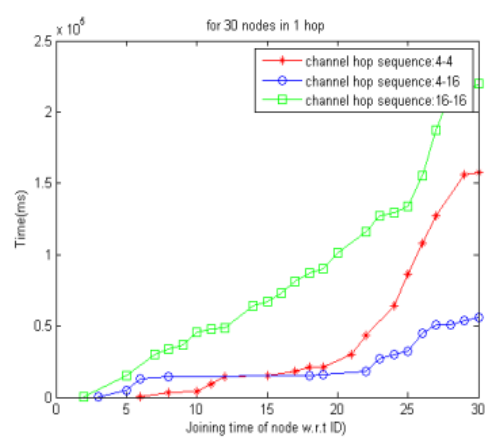

(a)

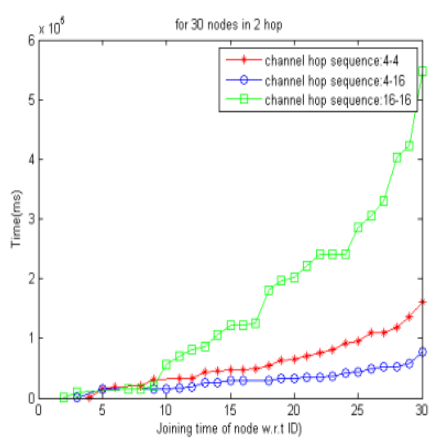

(b)

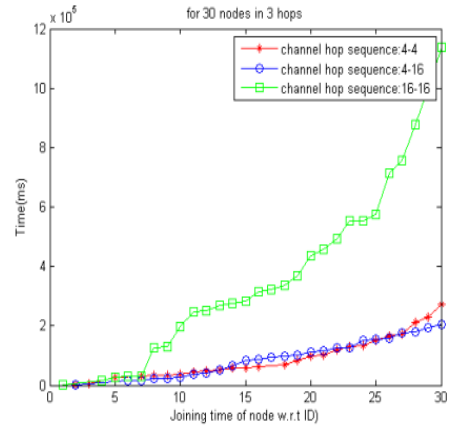

(c)

Fig. 10 Analysis of available channel hopping sequences in a(a) Single-ring network (b) Two ring network (c) Three ring network 


\section{REFERENCES}

[1] Elvis Vogli, Giuseppe Ribezzo, Luigi Alfredo Grieco, Gennaro Boggia Dipartimento di Elettrotecnica ed Elettronica, Politecnico di Bari," Fast Join and Synchronization Schema in the IEEE 802.15.4e MAC", in IEEE Wireless Communications and Networking Conference (WCNC), 2015.

[2] Domenico De Guglielmo1, Simone Brienza1, Giuseppe Anastasi1,' IEEE 802.15.4e: a Survey", Volume 88, Issue C, August 2016.

[3] T. Watteyne, M. Palattella, L. Grieco,'RFC 7554”, IETF. [Online]. Available: https://tools.ietf.org/html/rfc 7554

[4] Rainer Drath, Alexander Horch, "Industrie 4.0: Hit or Hype?" in IEEE industrial electronics magazine, June 2014.

[5] Peng Du, C Dr.George Roussos, "Adaptive Time Slotted Channel Hopping for Wireless Sensor Network", December 2012

[6] Magnus Jonsson, Kristina Kunert, "Towards Reliable Wireless Industrial Communication with Real-Time Guarantees" in IEEE transactions on industrial informartics, vol.5, no.4, nov.2009.

[7] Wei Shen, Tingting Zhang, Filip Barac, Mikael Gidlund, "PriorityMAC: A Priority-Enhanced MAC Protocol for Critical Traffic in Industrial Wireless Sensor and Actuator IEEE 2015 , vol.10,no.1, Feb.2014.
[8] Ivan muller, Joao cesar netto, Carlos Eduardo Pereira,"wirelessHART field devices", December 2011.

[9] Stig Petersen, Simon carlsen,"wirelessHART vs.ISA100.11a,’December 2011.

[10] Borja Martinez, Xavier Vilajosana, Member, IEEE, Fabien Chraim, Ignasi Vilajosana, and Kristofer S. J. Pister," When Scavengers Mee Industrial Wireless" Ieee Transactions On Industrial Electronics, Volume: 62, Issue: 5, May 2015.

[11] Vebhi C. Gungor, Gerhard P. Hancke, "Industrial Wireless Sensor Networks: Challenges, Design Principles, and Technical Approaches", IEEE transactions on industrial electronics, VOL. 56, NO. 10, October 2009.

[12] Ted H. Szymanski, "Supporting Consumer Services in a Deterministic Industrial Internet Core Network”, IEEE Communications Magazine, June 2016.

[13] Brendan Galloway and Gerhard P. Hancke, "Introduction to Industrial Control Networks", IEEE Communications Surveys \& Tutorials, Vol. 15, No. 2, Second Quarter 2013. 